\title{
Effect of once weekly oral levothyroxine therapy
}

Correspondence should be addressed to A del Toro-Diez

Email

andrea.deltoro@upr.edu

\section{Summary}

Primary hypothyroidism is one of the most common endocrine disorders with widely available treatment. A minority of patients remain with uncontrolled hypothyroidism despite therapy. The objective of this case series was to demonstrate that medication non-adherence, rather than malabsorption, should be sought as the most common cause of unsuppressed TSH levels in patients receiving treatment for this condition. Non-adherence is often considered as a diagnosis of exclusion. Nonetheless, a diagnosis of malabsorption requires a more extensive workup, including imaging and invasive procedures, which increase healthcare costs and burden to the patient. The findings of this study allow for a cost-effective approach to uncontrolled hypothyroidism.

\section{Learning points:}

- Medication non-adherence is a common cause of insuppressible TSH levels.

- Once weekly levothyroxine is an alternative approach to non-compliant patients.

- Assessing compliance is more cost-effective and less burdensome than testing for malabsorption.

\section{Background}

Hypothyroidism is one of the most common endocrine disorders seen among Puerto Ricans, and levothyroxine (LT4) has been considered the gold-standard treatment for many years. A study by Gonzalez-Rodriguez et al. found a prevalence of hypothyroidism of $24.2 \%$ in a sample of 400 postmenopausal women in Puerto Rico (1).

Levothyroxine must be taken on an empty stomach for adequate absorption in the small intestine. This may compromise adherence to therapy in some patients who may not be willing to undergo fasting. The American Thyroid Association (ATA) guidelines on hypothyroidism suggest that taking levothyroxine at bedtime (3 or more $\mathrm{h}$ after the evening meal) may be the next best choice for those who not compliant with the fasting technique (2). A randomized crossover trial by Grebe et al. demonstrated that once weekly levothyroxine therapy was also a safe and effective method for treating hypothyroidism, without any associated toxicities (3). \footnotetext{
open 2 Access International License.
}

Although it is agreed by many that non-compliance is the most common cause of TSH non-supressibility in hypothyroid patients, this is a diagnosis of exclusion, and a careful review for possible medication interactions should be performed. For example, phenytoin, phenobarbital, carbamazepine, and rifampin are known to increase levothyroxine clearance. On the other hand, impaired absorption is seen with gastrointestinal disorders, such as Helicobacter pylori-related gastritis, Celiac disease, and Chron's disease, and certain medications, such as proton pump inhibitors, bile acid sequestrants, calcium carbonate, and ferrous sulfate (4). Despite the possible struggles in management, thyroid extracts are not recommended by the ATA for hypothyroid patients, due to potential safety concerns.

We present four cases of hypothyroid patients receiving high doses of either LT4 or LT4 plus liothyronine (LT3) and a non-suppressible TSH, despite reported (c) 2021 The authors $\begin{array}{r}\text { https://edm.bioscientifica.com/ } \\ \text { Published by Bioscientifica Ltd }\end{array}$ Downloaded from Bioscientifica.com at 04/26/2023 12:06:11AM 
medication adherence. LT4 was administered at a dose of $1.6 \mu \mathrm{g} / \mathrm{kg} \times 7$, once weekly, as used by Jayakumari et al. (5) and Walker et al. (6). Currently, there are various protocols and no well-established standards for the levothyroxine absorption test, for which a 6-week weight-based approach was used. Data were collected using the same laboratory assay, technician, and facilities. Our patients were overweight or obese and denied weight loss, steatorrhea, nausea, or vomiting. Since no signs or symptoms of malabsorption were present, we believed that performing an extensive workup for this condition before testing for non-compliance would have been unnecessary and money- and time-consuming. Our study demonstrates the cost-effectiveness of testing for non-compliance before malabsorption. Nonetheless, this test may also be useful to identify concurrent malabsorptive problems.

\section{Case presentation}

Our first patient was a 52-year-old woman, weighing 62.5 $\mathrm{kg}$, with persistently uncontrolled primary autoimmune hypothyroidism despite receiving Levoxyl $425 \mu \mathrm{g}$ daily, for a total weekly dose of $2975 \mu \mathrm{g}$. This added up to a prestudy dose of $6.8 \mu \mathrm{g} / \mathrm{kg}$. Hypothyroidism was diagnosed 5 years prior to the study. Her baseline TSH concentration was $56.33 \mathrm{mIU} / \mathrm{L}$ and FT4 was $0.04 \mathrm{ng} / \mathrm{dL}$. She reported constipation, fatigue, cold intolerance, and a physical exam was remarkable for delayed deep tendon reflexes. She received a supervised total weekly dose of $700 \mu \mathrm{g}$ of Synthroid, as calculated by weight, for a total of 6 weeks. At the end of the 6 weeks period, her TSH concentration was $0.75 \mathrm{mIU} / \mathrm{L}$ and FT4 was $1.47 \mathrm{ng} / \mathrm{dL}$.

Our second patient was a 49-year-old obese male, weighing $122 \mathrm{~kg}$, with uncontrolled primary autoimmune hypothyroidism, taking $375 \mu \mathrm{g}$ daily, for a total weekly dose of $2625 \mu \mathrm{g}$. He had a pre-study dose of $3.1 \mu \mathrm{g} / \mathrm{kg}$. Diagnosis was made approximately 10 years prior to the study. His baseline laboratories were remarkable for a TSH of $12.8 \mathrm{mIU} / \mathrm{L}$ and a FT4 of $0.66 \mathrm{ng} / \mathrm{dL}$. Patient was asymptomatic. Our patient received a supervised total weekly dose of $1400 \mu \mathrm{g}$ of Synthroid for 6 consecutive weeks. At the end of the study period, his TSH was $0.72 \mathrm{mIU} / \mathrm{L}$ and FT4 was $1.42 \mathrm{ng} / \mathrm{dL}$.

Our third patient was a 46-year-old obese female, weighing $125 \mathrm{~kg}$, with uncontrolled primary autoimmune hypothyroidism diagnosed 8 years ago, taking Synthroid $550 \mu \mathrm{g}$ daily, for a total weekly dose of $3850 \mu \mathrm{g}$. She had a pre-study dose of $4.4 \mu \mathrm{g} / \mathrm{kg}$ of LT4. Her baseline TSH was $4.92 \mathrm{mIU} / \mathrm{L}$ and FT4 was $1.19 \mathrm{mIU} / \mathrm{L}$. Patient was asymptomatic. She received a supervised total weekly dose of $1400 \mu \mathrm{g}$ of Synthroid for 6 weeks. In week 3, laboratory results showed improvement in her thyroid function tests, with a TSH of $3.9 \mathrm{mIU} / \mathrm{L}$ and a FT4 of $2.27 \mathrm{ng} / \mathrm{dL}$, which are above the reference range. Then, in week 6 , an elevated TSH and normal FT4 were observed.

Our fourth patient was a 46-year-old obese female, weighing $103 \mathrm{~kg}$, with uncontrolled primary autoimmune hypothyroidism diagnosed 7 years ago, despite taking Levoxyl $200 \mu \mathrm{g}$ daily, for a total weekly dose of $1400 \mu \mathrm{g}$. She was receiving a pre-study dose of $2.0 \mu \mathrm{g} / \mathrm{kg}$ of LT4. Her baseline TSH concentration was $17.42 \mathrm{mIU} / \mathrm{L}$ and FT4 was $0.78 \mathrm{ng} / \mathrm{dL}$. At the moment of the study, she only reported fatigue and a physical exam was normal. She received a supervised total weekly dose of $1150 \mu \mathrm{g}$ of Synthroid for the first 2 weeks. The dose was then reduced to $950 \mu \mathrm{g}$ once weekly for the remainder of the study, due to the over suppression of TSH $(0.23 \mathrm{mIU} / \mathrm{L})$ and elevation of FT4 (2.77 $\mathrm{ng} / \mathrm{dL}$ ) with the initial calculated dose. At the end of the study, her TSH and FT4 levels were normal.

\section{Treatment}

Patients were treated with oral levothyroxine calculated as $1.6 \mu \mathrm{g} / \mathrm{kg}$. The medication was provided as a single weekly dose, on Thursdays, for a total of 6 weeks.

\section{Outcome and follow-up}

The first and second patients in our case series demonstrated suppressibility of serum TSH values and normalization of FT4 levels with once weekly levothyroxine therapy by the end of the study period at week 6 .

The third patient described in our case series was able to achieve suppressible serum TSH concentrations by week 3 , together with increased levels of FT4, which demonstrated adequate levothyroxine absorption. Afterward, her serum TSH concentration increased and remained above the normal range. This is attributed to an interruption in the continuity of the study due to natural disasters (hurricanes Irma and Maria) in Puerto Rico during the time of the levothyroxine absorption test.

The fourth patient described in the case series exhibited over suppression of serum TSH by week 3, together with FT4 elevation, even though levothyroxine therapy was initially started at a dose calculated by weight. The dose was adjusted accordingly, and serum TSH and FT4 levels normalized by the end of the follow-up period at week 6 . 


\section{Discussion}

Medication non-adherence affects approximately 40-50\% of patients with chronic diseases. Adherence to medical therapy is defined in most studies as taking $80 \%$ or more of the prescribed medication doses. Barriers to medication adherence include lack of patient motivation, depression, cognitive impairment, low educational level, drug or alcohol use, complexity, and costs of treatment, fear of side effects, poor physician-patient relationship, and asymptomatic disease (7).

Table 1 summarizes the baseline characteristics of our patients. As demonstrated in Figs 1 and 2, there is an overall trend toward suppressibility of serum TSH and an increase in FT4 after 6 consecutive weeks of once weekly levothyroxine bolus treatment, as determined by the patient's weight. All of the patients' calculated levothyroxine doses were less than the doses they had been receiving prior to the study and all patients had longstanding primary hypothyroidism, presumed to be of autoimmune etiology, given no history of thyroid surgery or radioiodine therapy. All patients tolerated the once weekly levothyroxine bolus without adverse effects. A workup for malabsorption was not performed in these patients in order to be cost-effective, given most patients were overweight or obese and they had no signs or symptoms suggesting this as a possible etiology.

The first and second patients in our case series demonstrated suppressibility of serum TSH values and normalization of FT4 levels with once weekly levothyroxine therapy. These results show that initial TSH nonsuppressibility in these patients was clearly due to noncompliance with the medication regimen.

The third patient described in our case series was able to achieve suppressible serum TSH concentrations by week 3 , together with increased levels of FT4, which demonstrated adequate levothyroxine absorption. Afterward, her serum TSH concentration increased and remained above the normal range. This is attributed to an interruption in the continuity of the study due to natural disasters (hurricanes Irma and Maria) in Puerto Rico during the time of the levothyroxine absorption test.
The fourth patient described in the case series exhibited over suppression of serum TSH by week 3, together with FT4 elevation, even though levothyroxine therapy was initially started at a dose calculated by weight. This strongly suggests that malabsorption is not present. The dose was adjusted accordingly, and serum TSH and FT4 levels were normalized by the end of the follow-up period. This demonstrates that when taken correctly and with adequate compliance, levothyroxine therapy is indeed capable of restoring euthyroidism.

We conclude that non-adherence to medical therapy was the most likely reason for non-suppressibility of serum TSH values in these patients who had been taking much larger doses of levothyroxine than merited by weight. In most cases, a thorough medical history and physical examination, including medication history and BMI, are enough to determine the cause for persistent uncontrolled hypothyroidism despite large doses of thyroxine replacement.

This condition in which the patient denies poor adherence is termed by some authors, pseudomalabsorption and often results in challenges in diagnosis and management. A complete malabsorption workup often includes a battery of blood tests and stool samples, together with radiologic imaging, endoscopy, and breath tests to determine a specific etiology. This process may be time-consuming, invasive, and results in higher costs and stress to the patient in the phase of an uncertain diagnosis. We believe that the evaluation of pseudomalabsorption with a supervised weekly levothyroxine bolus test is simple and cost-effective and should be done before a complete malabsorption workup unless the patient's symptoms clearly indicate otherwise. Gonzales et al. demonstrated the effectiveness of the levothyroxine absorption test in distinguishing malabsorption from pseudomalabsorption in patients with hypothyroidism, with 12 out of 13 patients proving to have non-adherence with therapy (8). Rangan et al. also demonstrated two case reports of patients who had uncontrolled hypothyroidism due to suspected noncompliance with treatment, but then achieved normal TSH levels after therapy was changed to weekly levothyroxine (4).

Table 1 Baseline characteristics of patients.

\begin{tabular}{|c|c|c|c|}
\hline Patient & $\begin{array}{c}\text { Age at } \\
\text { diagnosis }\end{array}$ & Sex & Weight (kg) \\
\hline 1 & 47 & Female & 62.5 \\
\hline 2 & 39 & Male & 122 \\
\hline 3 & 38 & Female & 125 \\
\hline 4 & 39 & Female & 103 \\
\hline
\end{tabular}

\begin{tabular}{llc}
$\begin{array}{l}\text { Medication regimen } \\
\text { pre-test }\end{array}$ & & $\begin{array}{c}\text { Baseline TSH } \\
(\mathrm{mlU} / \mathrm{L})\end{array}$ \\
\hline Levoxyl at $6.8 \mu \mathrm{g} / \mathrm{kg}$ & & 56.33 \\
Synthroid at $3.1 \mu \mathrm{g} / \mathrm{kg}$ & & 12.8 \\
Synthroid at $4.4 \mu \mathrm{g} / \mathrm{kg}$ & & 4.92 \\
Levoxyl at $2 \mu \mathrm{g} / \mathrm{kg}$ & & 17.4 \\
\hline
\end{tabular}

Signs/symptoms

Constipation, fatigue, cold intolerance, delayed deep tendon reflexes

Asymptomatic Asymptomatic Fatigue 
TSH VALUES $(\mu \mathrm{IU} / \mathrm{ml})$

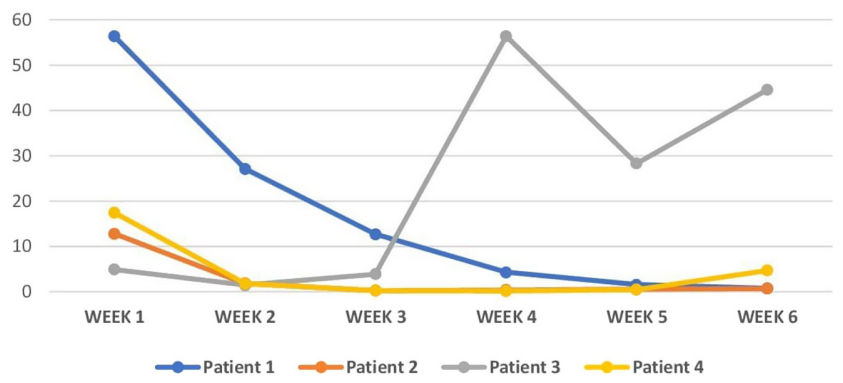

Figure 1

Weekly TSH values per patient.

Because the elimination half-life of thyroxine is approximately 7 days, it is plausible to administer once weekly thyroxine therapy in order to restore euthyroidism. Once weekly dosing helps maintain euthyroidism because intermittent administration of thyroxine may suppress antibody levels (9). Another mechanism is that at the end of the weekly treatment, when FT4 levels are considerably lower, there is more peripheral conversion of $\mathrm{T} 4$ to the metabolically active T3, whereas conversion of T4 to the metabolically inactive rT3, is decreased (3). Our results also aid us in avoiding the use of more complex replacement therapies that may result in higher costs and not necessarily control thyroid function tests. It has been demonstrated that the use of generic is as effective as brand-name levothyroxine in controlling thyroid dysfunction (10). Nonetheless, it is recommended that patients adhere to the same therapy throughout the treatment period, as the use of different formulations may impact the control of thyroid disease because they often have different bioavailability.

This case series demonstrates the cost-effectiveness of testing for pseudomalabsorption as an initial step. Using our first patient as a reference, before this study she was receiving $425 \mu \mathrm{g}$ daily of LT4, resulting in a weekly cost of $\$ 12.46$ and a monthly cost of $\$ 53.40$, if calculated using one LT4 $300 \mu \mathrm{g}$ pill and a $125 \mu \mathrm{g}$ pill. After the study, she needed $700 \mu \mathrm{g}$ weekly of LT4, for a weekly cost of $\$ 2.71$ and a monthly cost of $\$ 10.84$. This was calculated using one $300 \mu \mathrm{g}$ pill and two $200 \mu \mathrm{g}$ pills. Medication compliance should be reinforced, and this will subsequently result in a significant reduction in cost with regards to pills, laboratory evaluation, and healthcare visits.

Potential limitations of our study are a small number of patients, one patient had an interruption in the continuity of treatment, and patients were not followed after the termination of the study to determine persistent compensation of thyroid function, as further follow-up was provided by Endocrinologists outside our clinics.

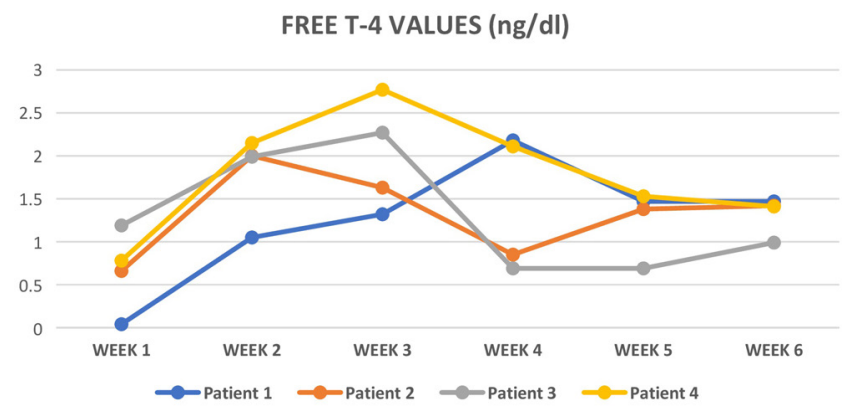

Figure 2

Weekly free $\mathrm{T} 4$ values per patient.

Strengths of the study include consistency of results and that the same laboratory assay, technician, and facilities were used for the duration of the study. Further studies could be performed to determine the effect of once weekly dosing on lipid homeostasis and quality of life.

\section{Declaration of interest}

The authors declare that there is no conflict of interest that could be perceived as prejudicing the impartiality of the research reported.

\section{Funding}

This research did not receive any specific grant from any funding agency in the public, commercial, or not-for-profit sector.

\section{Patient consent}

Written consent was obtained from patients.

\section{Author contribution statement}

Dr Del Toro-Diez was involved in the literature review, manuscript writing, and revision. Dr Ernesto Solá-Sánchez and Dr Michelle Mangual-García are Endocrinologists who were involved in patient care and manuscript revision.

\section{References}

1 Gonzalez-Rodriguez LA, Felici-Giovanini ME \& Haddock L. Thyroid dysfunction in an adult female population: a population-based study of Latin American Vertebral Osteoporosis Study (LAVOS) - Puerto Rico site hypothytoidism in LAVOS-Puerto Rico site. Puerto Rico Health Sciences Journal 201332 57-62.

2 Jonklaas J, Bianco AC, Bauer AJ, Burman KD, Cappola AR, Celi FS, Cooper DS, Kim BW, Peeters RP, Rosenthal MS et al. Guidelines for the treatment of hypothyroidm. Thyroid 201424 1670-1751. (https://doi. org/10.1089/thy.2014.0028)

3 Grebe SK, Cooke RR, Ford HC, Fagerström JN, Cordwell DP, Lever NA, Purdie GL \& Feek CM. Treatment of hypothyroidism with once weekly thyroxine. Journal of Clinical Endocrinology and Metabolism 199782 870-875. (https://doi.org/10.1210/jcem.82.3.3830) 
4 Rangan S, Tahrani AA, Macleod AF \& Moulik PK. Case Report: Once weekly thyroxine treatment as a strategy to treat non-compliance. $B M J$ 200783 e1-e2. (https://doi.org/10.1136/pgmj.2007.060244)

5 Jayakumari C, Nair A, Puthiyaveettil Khadar J, Das DV, Prasad N, Jessy SJ, Gopi A \& Guruprasad P. Efficacy and safety of once-weekly thyroxine for thyroxine-resistant hypothyroidism. Journal of the Endocrine Society 20193 2184-2193. (https://doi.org/10.1210/ js.2019-00212)

6 Walker JN, Shillo P, Ibbotson V, Vincent A, Karavitaki N, Weetman AP, Wass JA \& Allahabadia A. A thyroxine absorption test followed by weekly thyroxine administration: a method to assess non-adherence to treatment. European Journal of Endocrinology 2013168 913-917. (https://doi.org/10.1530/EJE-12-1035)
7 Kleinsinger F. The unmet challenge of medication nonadherence. Permanente Journal 201822 18-33. (https://doi.org/10.7812/TPP/18-033)

8 Biondi B. A report of the Mayo clinic experience on the levothyroxine absorption test. Clinical Thyroidology 202032 6-8. (https://doi. org/10.1089/ct.2020;32.6-8)

9 Altuntas F, Uysal AR, Corapcioglu D, Erol C. Twice weekly LT4 for the treatment of primary hypothyroidism. Turkish Journal of Endocrinology and Metabolism 20041 25-34.

10 Brito JP, Ross JS, Sangaralingham L, Dutcher SK, Graham DJ, Wang Z, Wu Y, Yao X, Smallridge RC, Bernet V et al. Comparative effectiveness of generic vs brand-name levothyroxine in achieving normal thyrotropin levels. JAMA Network Open 20203 e2017645. (https://doi. org/10.1001/jamanetworkopen.2020.17645)

Received in final form 20 May 2021

Accepted 7 June 2021 